\title{
Is genetic testing required in a child with an ovarian germ cell tumour?
}

\author{
Małgorzata Nowak1, Agata Pastorczak1, Hanna Moczulska², Dobromiła Barańska, \\ Karolina Krajewska' ${ }^{1}$ Elżbieta Sałacińska-Łoś', Wojciech Młynarski', Joanna Trelińska' \\ 'Department of Paediatrics, Oncology, and Haematology, Medical University of Lodz, Lodz, Poland \\ ${ }^{2}$ Department of Clinical Genetics, Medical University of Lodz, Lodz, Poland \\ ${ }^{3}$ Department of Paediatric Radiology, Medical University of Lodz, Lodz, Poland \\ ${ }^{4}$ Department of Paediatric Surgery, Medical University of Lodz, Lodz, Poland
}

\section{ABSTRACT}

In children the most commonly diagnosed ovarian neoplasms are germ cell tumours. The group that shows a particularly high risk of developing gonadal neoplasms are patients with complete gonadal dysgenesis with 46,XY karyotype (CGD, XY; Swyer syndrome).

A 12-year-old female patient was diagnosed with a mixed germ cell tumour located in the left ovary. The patient underwent chemotherapy and was provided with medical care at a genetic outpatient clinic. The secondary diagnosis of CGD, 46,XY resulted in the child being qualified for elective right-side gonadectomy. Imaging examinations that preceded the surgical procedure showed a cyst in the right ovary. It was resected together with the tumour, which was identified as undifferentiated pleomorphic sarcoma of giant cell type. The child has been regularly assessed at the oncological outpatient clinic, in the disease remission, for nine months since the second cancer was diagnosed.

KEY WORDS:

genetic predisposition, Swyer syndrome, genetic test, ovarian germ cell tumour, complete gonadal dysgenesis.

\section{INTRODUCTION}

In the paediatric population and young adults, the majority of lesions diagnosed in the area of the ovaries are benign. Primary ovarian cancer develops only in about $1.5 \%$ of cases [1], whereas malignant germ cell tumours account for $75 \%$ of ovarian neoplasms arising in the first 20 years of life and $15 \%$ of all neoplasms diagnosed in young individuals aged 15-19 years. These neoplasms are characterised by good prognosis associated with a survival probability reaching from $89 \%$ to $98 \%$ [2]. Gonadoblastoma is a potentially malignant mixed germ cell tumour that develops particularly frequently as a disorder secondary to dysgenetic gonads. If untreated, the neoplasm may transform into dysgerminoma, i.e. a tumour of an undeniably aggressive nature $[3,4]$.

The group exposed to the highest risk of gonadal neoplasms are patients with disorders of sex development (DSD) [5-9]. We may distinguish DSD associated with chromosome abnormalities (among others Turner syndrome 45,XX, Klinefelter syndrome 47,XXY, mixed gonadal dysgenesis $45, \mathrm{X} / 46, \mathrm{XY}$ ), DSD involving a $46, \mathrm{XY}$ male karyotype (associated with disorders of testicles development or defects in biosynthesis or function of androgens), and those involving a 46,XX female karyotype (associated with disorders of ovary development or an increased level of androgens) $[6,10]$.

\section{ADDRESS FOR CORRESPONDENCE:}

Joanna Trelińska, Department of Paediatrics, Oncology, and Haematology, Medical University of Lodz, 36/50 Sporna St., 91-738 Lodz, Poland, ORCID: 0000-0003-1484-4274, e-mail: joanna.trelinska@umed.lodz.pl 
The genetic predisposition to develop germ cell neoplasms also refers to carriers of germline mutations within the WT1 gene the phenotype of which corresponds to such conditions as Frasier syndrome, Denys-Drash syndrome, and WAGR syndrome [2]. However, the highest risk of malignant transformation occurs in patients suffering from complete gonadal dysgenesis (CGD) with 46,XY (Swyer syndrome), and it reaches 30-45\% throughout the lifetime and increasing even up to $80 \%$ in the fifth decade of life [3, 5, 7-9].

Swyer syndrome (SS) in approximately $10-20 \%$ is caused by a deletion in the DNA-binding region within the sequence of the sex-determining region Y (SRY) located in the short arm of Y (Yp11.3) chromosome $[6,11]$. In the remaining $80 \%$ of female patients presenting this syndrome the structure of the SRY gene is normal, and the disease occurrence results from mutations located in the other genes (ARX, ATRX, CBX2, DHH, DMRT1, GATA4, MAMLD1, MAP3K1, NROB1, NR5A1 SOX9, WNT4, WT1, WWOX, DAX-1), which determine the process of gonad differentiation or genetic defects that have not been identified yet $[3,6,7,11]$. In male individuals the development of an undifferentiated male gonad into a testicle results from the presence of a normally functioning $S R Y$ gene the expression of which activates production of testosterone by Leydig cells. Testosterone, in turn, stimulates the development of vas deferens and epididymides and anti-Müllerian hormone, which is responsible for the atrophy of the Müllerian ducts. In the case of abnormal function of the SRY gene, dysgenetic gonads are formed. They do not ensure a proper production of hormones, including secretion of $\mathrm{AMH}$, hence anatomical structures (i.e. the vagina, uterus, oviducts) derived from the Müllerian ducts develop in patients with the 46,XY karyotype $[6,11]$. Dysgenetic gonads do not show a typical ovarian structure and usually resemble connective tissue streaks $[5,11]$. In clinical terms, Swyer syndrome is characterised by the female phenotype, female external and internal genitalia, undeveloped secondary sex characteristics, primary amenorrhoea, and normal body growth [4]. Sex identification is usually female [6].

An early diagnosis of complete gonadal dysgenesis is difficult, and it is usually established in adolescence when the causes of primary amenorrhoea and a lack of breast development are sought $[3,4,6]$. For this reason, at the stage when a 46,XY karyotype is found, patients are often already diagnosed with tumours deriving from gonadal dysgenesis (approximately 55\%), mainly gonadoblastoma or dysgerminoma [4]. At present the only procedure that protects patients with the 46,XY karyotype against development of cancer is prophylactic gonadectomy $[3,5]$.

\section{CASE REPORT}

The clinical case concerns a 12 -year-old girl who presented at the provincial hospital due to abdominal pain of several days' duration. No significant diseases in her past medical history were revealed. Sexual maturity according to Tanner staging was assessed as Thel I, Ph I, Ax (-), with normal body height $(156 \mathrm{~cm}, 90-97 \%$ for age and sex). Based on the clinical picture and additional examinations, including abdominal ultrasound, which showed a periappendicular infiltrate, the patient was diagnosed with appendicitis and was qualified for surgery. During the procedure a tumour (size $20 \mathrm{~cm} \times 10 \mathrm{~cm} \times$ $10 \mathrm{~cm}$ ) was detected; it was bleeding intensively from the surface of several ruptures. The tumour was located along the body's midline arising from the left ovary, with the adjacent vermiform appendix showing inflammatory changes. The vermiform appendix and the left oviduct were removed. The day after the surgical procedure the marked level of $\alpha$-fetoprotein was significantly elevated $(2960.9 \mathrm{IU} / \mathrm{ml}$, with the normal range up to $5 \mathrm{IU} / \mathrm{ml})$, and the level of $\beta$ human chorionic gonadotropin ( $\beta-\mathrm{HCG}$ ) was normal $(0.46 \mathrm{mlU} / \mathrm{ml}$, with the normal range up to $5 \mathrm{IU} / \mathrm{ml})$. Because a germ cell tumour was suspected, the girl was referred to the Department of Paediatric Oncology and Haematology to undergo further diagnostic procedures and treatment. The diagnosis of an ovarian germ cell tumour of mixed type was confirmed based on the histopathological assessment. The tumour tissue was composed of elements of the yolk sac tumour and focally with elements of embryonal carcinoma with features of angioinvasion in small vessels of the serosa. Additionally, cancer cells in the peritoneal cavity fluid were also found. No distant metastases were observed in the patient. Based on the overall clinical picture, the patient was qualified for chemotherapy according to the TGM 95 protocol for malignant germ cell type tumours of high risk (VIP cycle: etoposide, ifosfamide, cisplatin). The markers were normalised after the second cycle, and the girl received four VIP cycles of chemotherapy. The patient achieved complete remission, and she was further monitored at an oncology outpatient clinic.

Due to a family history of cancer (bilateral breast cancer at the age of 45 years and endometrial carcinoma at the age of 50 years in the patient's maternal grandmother; endometrial carcinoma at the age of 50 years in the grandmother's sister; acute leukaemia diagnosed at the age of 30-50 years in three of her paternal grandfather's sisters; her grandfather's mother diagnosed with chronic lymphocytic leukaemia as an elderly person), the patient received genetic counselling. Sequencing of $B R C A 1$ and $B R C A 2$ genes was performed in the patient, obtaining a negative result with regard to the presence of mutations in the two genes. The genetic history of the patient's family is presented in Figure 1.

One year after the completion of the oncologic treatment (the patient was 13 years old then) due to delayed development of secondary sex characteristics (according to Tanner staging: Thel I, Ph I, Ax [-]) and amenorrhoea, the girl was admitted to hospital to undergo the recom- 


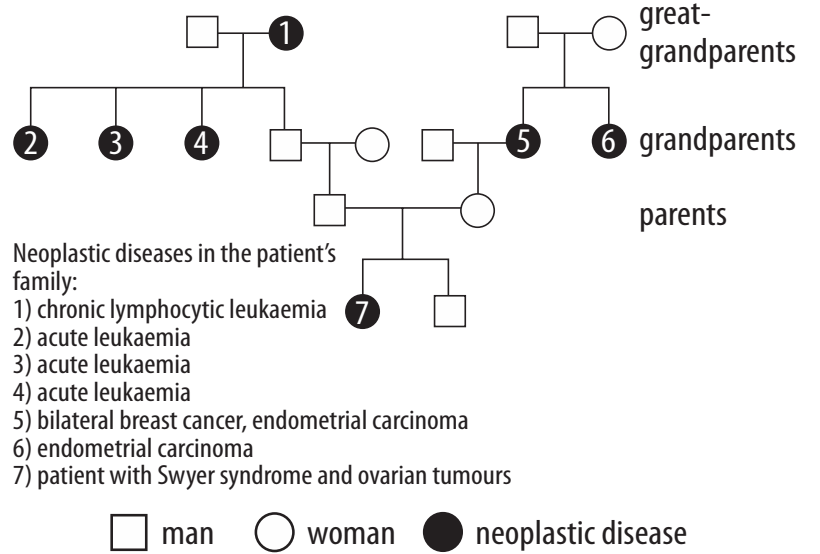

FIGURE 1. The genetic history of the patient's family

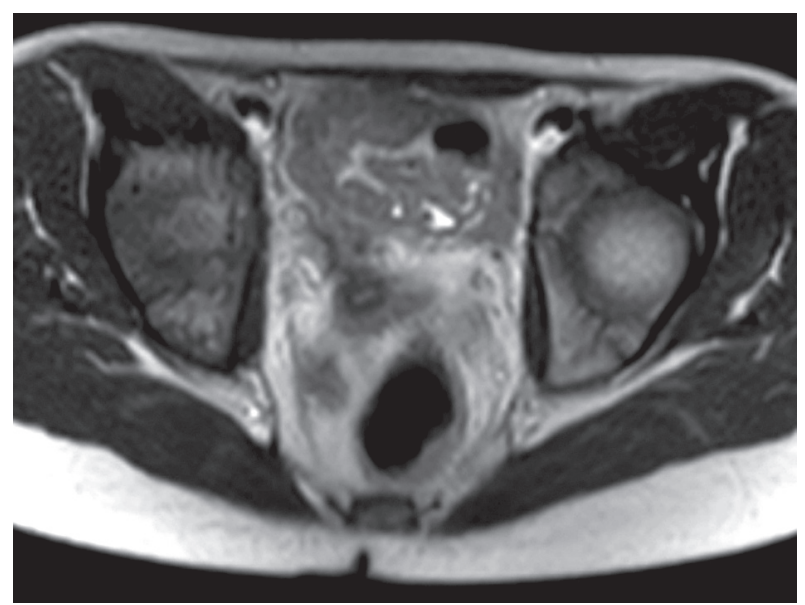

FIGURE 2. Magnetic resonance imaging of the pelvis performed one year after completion of chemotherapy: a small uterus was visualised, ovaries were not observed

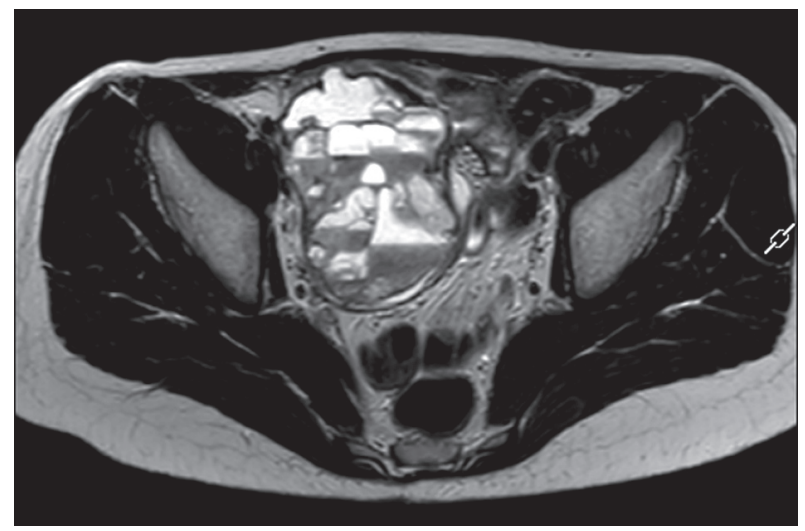

FIGURE 3. Magnetic resonance imaging of the pelvis performed before the second surgery; a multichamber cyst in the right ovary was visualised

mended imaging and hormonal tests. An ultrasound scan revealed a prepubertal uterus, with the body size of approx. $14 \mathrm{~mm} \times 25 \mathrm{~mm} \times 31 \mathrm{~mm}$, slightly larger than the cervix. The right ovary was poorly visible, with size approx. $8 \mathrm{~mm} \times 12 \mathrm{~mm} \times 22 \mathrm{~mm}$, with a few very tiny follicles. MRI examination confirmed the existing disease remission (Fig. 2). Hormone tests showed an elevated level of the follicle-stimulating hormone (FSH) $(93.90 \mathrm{mIU} / \mathrm{ml}$, normal range: $0.19-7.97 \mathrm{mIU} / \mathrm{ml})$, an elevated level of the luteinising hormone (LH) $(36.43 \mathrm{mIU} / \mathrm{ml}$, normal range: $0-15.26 \mathrm{mIU} / \mathrm{ml})$, and a decreased level of oestradiol ( $<10 \mathrm{pg} / \mathrm{ml}$, normal range: $7-97 \mathrm{pg} / \mathrm{ml}$ ). The patient was diagnosed with hypergonadotropic hypogonadism. Therefore, she was admitted to the endocrinology outpatient clinic and began hormonal replacement therapy.

In the view of new medical data, a karyotype of peripheral blood lymphocytes was tested, and the obtained result was $46, \mathrm{XY}$. The genetic test did not show any mutations in the SRY gene. Finally, the patient, at the age of 14 years (13 years and 9 months), was diagnosed with complete gonadal dysgenesis, which explained the girl's health problems. Due to a remarkably high risk of malignant transformation within the right ovary, right-sided elective gonadectomy was recommended.

The diagnosis, which was very difficult to accept because of its psychological aspect, caused a delay in the surgical procedure. During that time subsequent imaging examinations, including MRI, showed a growing multichamber cyst in the right ovary (Fig. 3). Tumour markers: AFP, $\beta$-HCG, and CA-125, determined regularly at that time, were within normal ranges. Finally, seven months after the patient had been recommended prophylactic gonadectomy, the patient presented to the Department of Surgical and Conservative Gynaecology to undergo the surgical procedure. Then the right ovary was removed, together with the tumour, size $6.5 \mathrm{~cm} \times 5.5 \mathrm{~cm} \times 5 \mathrm{~cm}$, the hypoplastic uterus and bilateral iliac lymph nodes. Histopathological examination revealed undifferentiated pleomorphic sarcoma - giant cell type, malignant fibrous histiocytoma (MFH), infiltrating the oviduct and the uterine corpus, with no metastases to the lymph nodes (Fig. 4). Oophorectomy was a macroscopic radical resection, whereas microscope radicality evaluation was not possible due to a lack of detailed macroscopic markers. In order to assess the disease progression, the following imaging examinations were performed: magnetic resonance imaging of the abdomen and pelvis (Fig. 5), computed tomography of the thorax, positron emission tomography - PET/CT, and bone scintigraphy. The results excluded any presence of distant metastases. Therefore, in compliance with the applicable treatment protocol for soft tissue sarcoma, the 2006 CWS guidance, oncologic treatment was abandoned.

The patient has been regularly assessed at oncologic, endocrine, and psychological outpatient clinics, remaining in disease remission, nine months after being diagnosed with the second cancer.

\section{DISCUSSION}

Complete gonadal dysgenesis with the 46,XY karyotype is a rare disease, occurring in one per 8000 births $[3,7]$. Patients with this syndrome are characterised by 


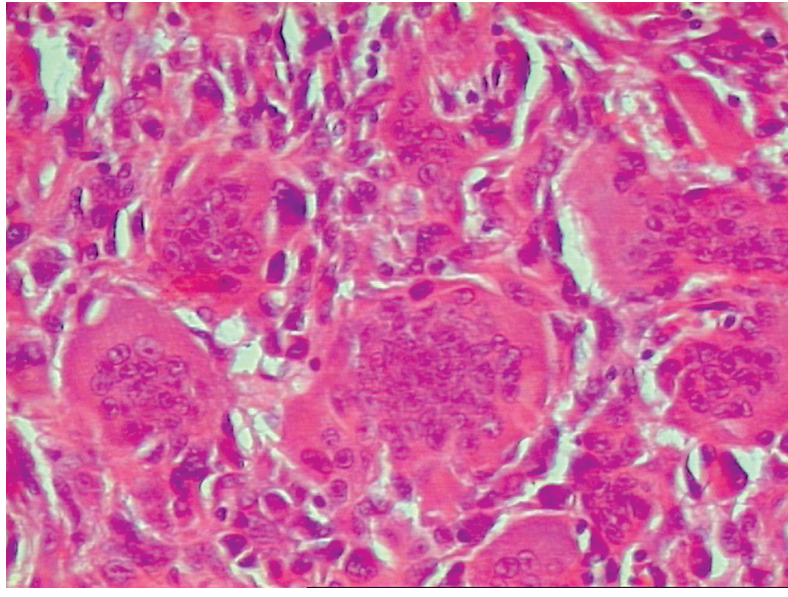

FIGURE 4. Undifferentiated pleomorphic sarcoma - giant cell type. Morphologically the tumour forms streaks of spindle cells with features of pleomorphism and presence of abundant multinucleated giant cells

a female phenotype with a male genotype $[6,11]$. Because the external genitalia look normal the disease usually remains undiagnosed for a long time. The most common indications for implementation of genetic diagnostic testing are primary amenorrhoea and a lack of development of secondary sex characteristics $[3,4,6,7]$. Imaging studies, including pelvic ultrasound or MRI, can be effective diagnostic tools for this group of patients, particularly in identifying hypoplastic uterus and streak gonads [6]. Genetic tests are preceded by laboratory diagnostics including determination of the level of sex hormones and, based on these results, hypergonadotropic hypogonadism is diagnosed (being the main cause of sexual development disorder) $[7,11]$. In many cases, patients with complete gonadal dysgenesis are diagnosed only in their adulthood; therefore, an option to identify early indicators of this condition would be particularly useful [4]. Carmen et al. suggest that FSH appears to be a valuable screening marker for diseases accompanied by gonadal dysgenesis [7].

The patient in the described clinical case, having completed therapy for a germ cell ovarian tumour, was referred to a genetic outpatient clinic, initially due to a suspicion of cancer genetic predisposition in her family. The family history was particularly focused on the probability of occurrence of mutations in BRCA1 and BRCA2 genes in the patient's family. Mutations in these genes predispose predominantly to ovarian cancer, and in the case of $B R C A 2$ also to acute lymphoblastic leukaemia, which was diagnosed in several of the girl's relatives [2, 12]. Molecular diagnostics including next-generation sequencing of the whole coding region of the two genes, excluded the presence of mutations. At that time, when the patient was only 12 years old, the lack of breast development and primary amenorrhoea were not considered as a pathological condition in terms of sexual maturity. Laboratory diagnostics was extended only when the patient reached the

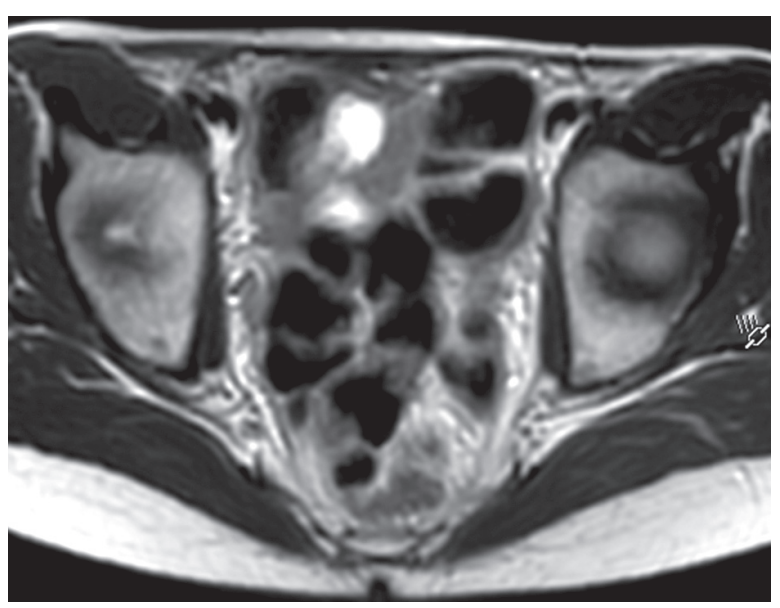

FIGURE 5. Magnetic resonance of the pelvis performed after the second surgery: intestinal loops visualised in the tumour bed

age of 13 years, and still no characteristics of sexual development were observed in the patient. Hormonal tests showed features of hypergonadotropic hypogonadism, which was an indication to perform further genetic testing. The diagnosis of Swyer syndrome was very important because of the high risk of malignant transformation in the area of dysgenetic gonads [5, 7-9].

So far, there have been no clear recommendations that indicate the necessity of performing genetic diagnostic tests in patients with gonadal germ-cell tumours. Only a diagnosis of gonadoblastoma suggests a possible genetic predisposition towards neoplasm, mainly in the course of gonadal dysgenesis related to disorders of sex development (DSD) or other syndromes of genetic predisposition towards neoplasm, such as Frasier syndrome or Denys-Drash syndrome [2]. In the discussed case, if the patient had undergone examinations and tests for DSD earlier, prophylactic gonadectomy might have prevented the development of cancer in her right ovary.

The diagnostic process of ovarian tumours is challenging and requires vast experience both from the medical team and diagnostic imaging specialists. At the time the first diagnosis of the ovarian tumour was established, the symptoms indicated acute appendicitis. The tumour in the right iliac fossa seen on ultrasound examination was regarded as a periappendicular infiltrate. These circumstances had an impact on delayed diagnosis of the neoplasm, including determination of basic oncological markers such as AFP, $\beta$-HCG, and CA-125. The mixed germ cell tumour diagnosed based on the histopathological examination is a rare neoplasm representing the group of germ cell tumours; however, it is regarded as one of those occurring in the course of CDG, 46,XY [6].

Undifferentiated pleomorphic sarcoma, previously defined as malignant fibrous histiocytoma (MFH), diagnosed in the right ovary was also latent in the analysed case. It was manifested by a multichamber cyst with no 
clinical symptoms, and on imaging examinations it resembled a benign lesion.

$\mathrm{MFH}$ is a rare tumour occurring mainly in adults aged between 50 and 70 years. It is usually located in the lower limbs and the retroperitoneal space. It derives from soft tissues; however, it may also affect bones. The first sign of the condition is most frequently a growing painless lump of soft tissue. At present the treatment involves a radical surgery including a resection margin of healthy tissue [13, 14]. However, several cases describing the occurrence of the neoplasm in ovaries of adult individuals were noted [15-18].

In children $\mathrm{MFH}$ is an extremely rare neoplasm, and its course and outcome are still unknown [1]. Single cases reported in the literature describe occurrence of the tumour in various locations in children, among others in the retroperitoneal space, skin, parotid salivary glands, and cranial bones [14, 19-21]. Incesoy-Özdemir et al. analysed the case of an 11-year-old boy who was diagnosed with MFH as a secondary neoplasm following treatment of acute lymphoblastic leukaemia [19].

Undifferentiated pleomorphic sarcoma does not correspond to the clinical picture of Swyer syndrome. In view of currently available data on this condition it may be hypothesised that in our patient it was a secondary neoplasm induced by chemotherapy in a child presenting a genetic predisposition towards neoplasms.

At present the treatment of CDG, 46,XY involves prophylactic bilateral laparoscopic gonadectomy as the only effective method preventing development of cancer in the future $[3,5,6]$. It is necessary to provide a regular endocrine assessment and introduce hormonal replacement therapy to ensure normal development of sex characteristics and induce menstrual bleeding $[3,6]$. Professional psychological counselling helps patients and their families to accept the disease. Diagnosing CDG, 46,XY does not exclude maternity either. Methods such as assisted reproductive technology, including oocyte donation, give a chance for having healthy offspring [4].

\section{CONCLUSIONS}

In conclusion, it should be emphasised that a diagnosis of disorders of sex development predisposing towards gonadal neoplasms is difficult and is often delayed. The first symptom of disorders of sex development may be a germ cell tumour. Therefore, in such a case, laboratory diagnostics should be implemented, including sex hormones, and recommendations for genetic consultation should be considered. Early prophylactic gonadectomy in a patient with DSD prevents the development of neoplastic disease in dysgenetic gonads.

\section{DISCLOSURE}

The authors declare no conflict of interest.

\section{REFERENCES}

1. Renaud EJ, Sømme S, Islam S, et al. Ovarian masses in the child and adolescent: An American Pediatric Surgical Association Outcomes and Evidence-Based Practice Committee systematic review. J Pediatr Surg 2019; 54: 369-377.

2. Goudie C, Witkowski L, Vairy S, et al. Paediatric ovarian tumours and their associated cancer susceptibility syndromes. J Med Genet 2018; 55: 1-10.

3. Keskin M, Şavaş-Erdeve S, Kurnaz E, et al. Gonadoblastoma in a patient with $46, \mathrm{XY}$ complete gonadal dysgenesis. Turk J Pediatr 2016; 58: 538-540.

4. Milewicz T, Mrozińska S, Szczepański W, et al. Dysgerminoma and gonadoblastoma in the course of Swyer syndrome. Pol J Pathol 2016; 67: 411-414.

5. McCann-Crosby B, Gunn S, Smith EO, et al. Association of immunohistochemical markers with premalignancy in Gonadal Dysgenesis. Int J Pediatr Endocrinol 2015; 2015: 14.

6. Jung EJ, Im DH, Park YH, et al. Female with 46, XY karyotype. Obstet Gynecol Sci 2017; 60: 378-382.

7. Capito C, Arnaud A, Hameury F, et al. Dysgerminoma and gonadal dysgenesis: The need for a new diagnosis tree for suspected ovarian tumours. J Pediatr Urol 2011; 7: 367-372.

8. Looijenga LH, Hersmus R, de Leeuw BH et al. Gonadal tumours and DSD. Best Pract Res Clin Endocrinol Metab 2010; 24: 291-310.

9. Abacı A, Çatlı G, Berberoğlu M. Gonadal malignancy risk and prophylactic gonadectomy in disorders of sexual development. J Pediatr Endocrinol Metab 2015; 28: 1019-1027.

10. Bajszczak K, Szarras-Czapnik M, Słowikowska-Hilczer J. Disorders of sex development - new terminology and new recommendations for therapeutic management. Polish Sexology 2014; 12: 74-80.

11. Yesiltepe Multu G, Kirmizibekmez H, Aydin H, et al. Pure gonadal Dysgenesis (Swyer syndrome) due to microdeletion in the SRY gene: a case report. J Pediatr Endocrinol Metab 2015; 28: 207-210.

12. Diets IJ, Esmé Waanders E, Ligtenberg M, et. al. High yield of pathogenic germline mutations causative or likely causative of the cancer phenotype in selected children with cancer. Clin Cancer Res 2018; 24: 1594-1603.

13. Moradi A, Afsharfard A, Atqiaee K. Undifferentiated Pleomorphic Sarcoma Presenting as Abdominal Pain with a Pulsatile Mass. Case Rep Surg 2016; 8251043.

14. Rothman AE, Lowitt MH, Pfau RG. Pediatric cutaneous malignant fibrous histiocytoma. J Am Acad Dermatol 2000; 42: 371-373.

15. Dilek TUK, Dilek S, Pata O, et al. Malignant fibrous histiocytoma of the ovary: a case report. Int J Gynecol Cancer 2006; 16 (Suppl 1): 352-356.

16. Savitchi E, Rao S. Squamous Cell Carcinoma and Pleomorphic Sarcoma (MFH) Arising in a Mature Cystic Teratoma of the Ovary. Int J Gynecol Pathol 2012; 31: 443-446.

17. Stefanovic A, Stojnic J, Jeremic K, et al. Malignant fibrous histiocytoma of the ovary: a case report. Eur J Gynaecol Oncol 2012; 33: 236-239.

18. Kurtoglu E, Celik H, Kokcu A, et al. Undifferentiated pleomorphic sarcoma with focally rhabdomyosarcomatous differentiation of the ovary. Eur J Gynaecol Oncol 2016; 37: 401-403.

19. Incesoy-Özdemir S, Balkaya E, Ören AC, et al. A Rare Type of Secondary Cancer in a Child With Acute Lymphoblastic Leukemia: Malignant Fibrous Histiocytoma. J Pediatr Hematol Oncol 2014; 36: e121-124.

20. Căglar K, Güngör S, Akansoy S, et al. Successful treatment of retroperitoneal giant cell-type malignant fibrous histiocytoma in a 5-year-old boy. Turk J Pediatr 2007; 49: 307-311.

21. Chang RC, Dave SP, Robinson PG. Undifferentiated pleomorphic sarcoma of the parotid gland: a rare pediatric case. Head Neck 2008; 30: $970-973$. 